\title{
Risk Management for Digital Transformation and Big Data in Architecture Board: A Case Study on Global En- terprise
}

\author{
Yoshimasa Masuda*, Seiko Shirasaka ${ }^{\dagger}$, \\ Shuichiro Yamamoto ${ }^{\ddagger}$, Thomas Hardjono ${ }^{\S}$
}

\begin{abstract}
This study investigates the solutions incorporated by the Architecture Board in global healthcare enterprise (GHE) to mitigate architecture risks especially in Digital IT areas covering Big Data while implementing the "Adaptive Integrated EA framework," which can be applied in companies promoting IT strategy with Cloud/Mobile IT. The study proposes and verifies the strategic risk mitigation model for digital transformation covering Big Data in Architecture Board, at the same time as this study revealed the distribution of solutions across the architecture domains in Enterprise Architecture covering applications and technologies with Cloud/Mobile IT/Big Data/Digital IT to mitigate risks. An in-depth analysis of this distribution resulted in the definition of the effective strategy elements to mitigate risks and a practical guidance for companies that consider Risk Management for Digital Transformation while starting up the Architecture Board in Enterprise Architecture with IT strategy covering Digital IT related elements focusing on Big Data.
\end{abstract}

Keywords - Enterprise Architecture, Digital Transformation, Risk Management, Architecture Board, Big Data, Cloud Computing, Digital IT

\section{Introduction}

Many global corporations have encountered a variety of changes, such as progress of new technologies, globalization, shifts in customer needs, and new business models. Significant changes in cutting-edge IT technology due to recent developments in Cloud computing and Mobile IT (such as progress in big data technology) have emerged as new trends in information technology. Furthermore, major advances in the abovementioned technologies and processes have created a "Digital IT economy," bringing about both business opportunities and business risks and forcing enterprises to innovate or face the consequences [3]. Enterprise systems (ES) are complex application software packages that contain mechanisms that support the management of the whole enterprise and integrate all areas in its function [6, p.121]. Enterprise Archi-

\footnotetext{
* Keio Graduate School of System Design and Management, Yokohama, Japan

$\dagger$ Keio Graduate School of System Design and Management, Yokohama, Japan

* Graduate School of Information Science, Nagoya University, Nagoya, Japan

$\S$ Media Lab, Connection Science and Engineering, Massachusetts Institute of Technology, Boston, USA
} 
tecture (EA) should be effective because it contributes to the design of large integrated systems, which represents a major technical challenge toward the era of Cloud/Mobile IT/Big Data/Digital IT in Digital Transformation. From a comprehensive perspective, EA encompasses all enterprise artifacts, such as business, organization, applications, data, and infrastructure, to establish the current architecture visibility and future architecture/roadmap. On the other hand, EA frameworks need to embrace change in ways that adequately consider the emerging new paradigms and requirements affecting EA, such as enterprise Mobile IT/Cloud [1, 4].

Furthermore, considering the above background, the previous study proposed the "Adaptive Integrated EA framework," which should align with IT strategy promoting Cloud/Mobile IT/Digital IT, and verified this in the case study [23]. The solutions to architecture risks raised in the Architecture Board Review for Digital IT projects in global healthcare enterprise (GHE) are also investigated, where the abovementioned EA framework is built and practiced as the only case study of related up-to-date EA toward the era of Digital IT.

This paper is organized as follows: the next section presents the background of this study, followed by the description of research methodology and overview of the Architecture Board in the "Adaptive Integrated EA framework." The results and data analysis in the case study are presented, and the Solutions proposing the Risk Mitigation model are verified. The Strategy elements to mitigate Risks in Digital Transformation are then formulated. Finally, the limitations of the current study and directions for future research are outlined.

\section{Direction of Enterprise Architecture}

\subsection{Related Work}

In the past decade, EA has become an important method for modeling the correlation for overall images of corporate and individual systems. In ISO/IEC/IEEE42010:2011, architecture framework is defined as "principles, and practices for the architecture descriptions established within a specific domain of application and/or community." Furthermore, EA visualizes the current corporate IT / business landscape to promote a desirable future IT model [4]. It is not a simple support activity [1], and it offers many benefits to companies, such as coordination, communication and planning between business and IT, and reduction in the complexity of IT [20]. For the delivery of these benefits, EA frameworks need to cope with the emerging new paradigms such as Cloud computing or enterprise mobility [1].

Mobile IT computing is an emerging concept using Cloud services provided over mobile devices [16]. In addition, Mobile IT applications are composed of Web services. Many studies discuss the integration of EA with Service Oriented Architecture (SOA), except for Mobile IT. The SOA architecture pattern defines the four basic forms of business service, enterprise service, application service, and infrastructure service [19]. The OASIS, which is a public standards group [14], introduces an SOA reference model. Many organizations have invested in SOA as an approach to manage rapid change [5]. Meanwhile, attention has been focused on Microservices architecture, which allows rapid adoption of new technologies, such as Mobile IT applications and Cloud computing [17]. SOA and Microservice vary greatly from service characteristics perspective [19]. Microservice is an approach for dispersed systems that is defined by the two basic forms of functional services through an API layer and infrastructure services. Multiple Microservices cooperating to work together enable the implementation as a Mobile IT application [7].

For Cloud Computing, the NIST defined three cloud service models such as SaaS, PaaS, and 
IaaS [12]. PaaS is an IaaS platform that includes both system software and an integrated development environment. SaaS is a software application developed, implemented, and operated on a PaaS foundation. IaaS accommodates PaaS and SaaS by offering infrastructure resources, such as computing network storage memory through specific centers [12]. Many Mobile IT applications also operate with SaaS Cloud-based software [16]. The integration and relationship between EA and Cloud computing is discussed rarely in literature. Considering the recent dynamic moves in Cloud computing, it is necessary for companies to link the service characteristics of EA and Cloud computing [13]. The traditional approach takes months to develop an EA realizing a Cloud adoption strategy, and organizations will demand adaptive enterprise architecture to iteratively develop and manage an EA adaptive to the Cloud technology [11].

Moreover, according to previous research [15], when promoting Cloud/Mobile IT in a strategic manner, it is proposed as a good option that a company that applies TOGAF or FEAF can adopt the integrated framework with the Adaptive EA framework supporting elements of Cloud computing. Table 1 shows the overview of the above related works for EA, Cloud computing and Mobile IT as below.

Table 1: Overview of Related works for EA, Cloud and Mobile IT

\begin{tabular}{|c|c|c|}
\hline Research Categories & Highlights & References \\
\hline \multirow[t]{2}{*}{ EA general } & Definitions of an architecture framework & $\begin{array}{l}\text { [4] S. Buckl, F. Matthes, C. Schulz, and C. } \\
\text { M. Schweda,2010; } \\
\text { [1] A. Alwadain, E. Fielt, A. Korthaus, } \\
\text { and M. Rosemann, 2014; }\end{array}$ \\
\hline & Benefits of EA & $\begin{array}{l}\text { [20] T. Tamm, P. B. Seddon, G. Shanks, } \\
\text { and P. Reynolds, 2011; }\end{array}$ \\
\hline \multirow[t]{2}{*}{ Mobile IT } & Concepts of Mobile IT & $\begin{array}{l}\text { [16] K. Muhammad and M. N. A. Khan, } \\
2015\end{array}$ \\
\hline & Analysis of EA and Mobile IT & $\begin{array}{l}\text { [15] Y. Masuda, S. Shirasaka, and S. } \\
\text { Yamamoto, } 2016\end{array}$ \\
\hline \multirow[t]{3}{*}{ Cloud computing } & Definitions of Cloud computing & [12] A. Q. Gill,2015 \\
\hline & Relationships between EA and Cloud computing & $\begin{array}{l}\text { [13] K. M. Khan and N. M. Gan- } \\
\text { gavarapu, } 2009 \\
\text { [11] A. Q. Gill, S. Smith, G. Beydoun, } \\
\text { and V. Sugumaran, } 2014\end{array}$ \\
\hline & Analysis of EA and Cloud & $\begin{array}{l}\text { [15] Y. Masuda, S. Shirasaka, and S. } \\
\text { Yamamoto, } 2016\end{array}$ \\
\hline \multirow{4}{*}{ SOA and Microservices } & Definitions of SOA & [19] M. Richards, 2015; \\
\hline & Reference model of SOA & $\begin{array}{l}\text { [14] C. M. MacKenzie, K. Laskey, F. } \\
\text { McCabe, P. F. Brown, and R. Metz, } 2006\end{array}$ \\
\hline & Benefits of SOA & $\begin{array}{l}\text { [5] H. Chen, R. Kazman, and O. Perry, } \\
2014\end{array}$ \\
\hline & Characteristics of Microservices & $\begin{array}{l}\text { [17] S. Newman, } 2015 \\
\text { [19] M. Richards, } 2015 \\
\text { [7] B. Familiar, } 2015\end{array}$ \\
\hline
\end{tabular}

\subsection{Background with Big Data}

New computing trends require data with far greater volume, velocity, and variety than ever before. Big data is utilized in ingenious methods to predict customer buying behaviors, detect fraud 
and waste, analyze product opinion, and react quickly to changes in business conditions (a driving force behind new business opportunities) [24]. The term "big data" refers to data that is so large, it is difficult to process using currently-available IT systems. There is a growing opportunity for analysis, visualization, and distributed processing software to enable users to extract useful information from such data [3]. Sources of big data include the following.

- Corporate data in SQL databases

- Data in cloud-based SQL or NoSQL databases

- Data provided by social networks

- Data provided by sensors or object identifiers in the internet-of-things (IoT)

Big data applications may include visualization functionality for effective user presentation of analytical results. Furthermore, big data applications should leverage web services that make the results of their analyses available to other applications through APIs; objects in the IoT can be data generators [3].

Existing big data reference architectures have been shepherded by NIST, which helped create the big data interoperability framework, including a reference architecture volume. The strengths of a NIST reference architecture include strict vendor neutrality, while providing clear definitions of big data terminology across many domains [25]. Figure 1 shows this NIST Big Data Reference Architecture (NBDRA).

The NBDRA represents a big data system comprised of five logical components connected by interoperability interfaces (i.e., services). These include "System Orchestrator," "Data Provider," "Big Data Application Provider," "Big Data Framework Provider," and "Data Consumer." Moreover, two fabrics envelop those five components: "Management" and "Security and Privacy" [25].

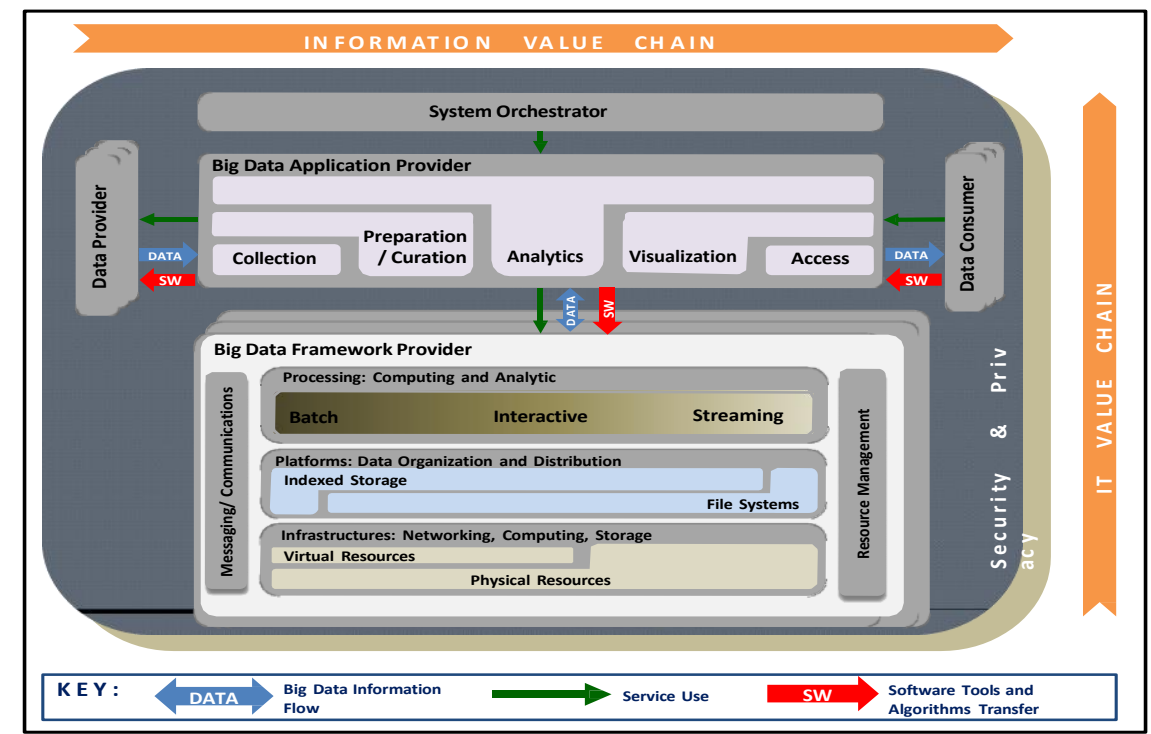

Figure 1: NIST Big Data Reference Architecture (NBDRA) (Source: US Department of Commerce, NIST Big Data Interoperability Framework: Reference Architecture Version 1, 2015)

The NBDRA is organized around two axes showing two big data value chains: the information (horizontal axis) and the IT (vertical axis). Along the information axis, value is created by data collection, integration, analysis, and application of these results into the value chain. Along 
the IT axis, value is created by offering networking, infrastructure, platforms, application tools, and other IT services for hosting and operating Big Data to support required data applications. The intersection of both axes is the Big Data Application Provider component, indicating that, in both value chains, data analytics and its implementation provide value to Big Data [25]. The "DATA" arrows in Figure 1 show the flow of data between the main components. The "SW" arrows show transfer of software tools for processing Big Data. The "Service Use" arrows show software programmable interfaces [25].

LinkedIn, for example, collects data from users and offers services, such as skill endorsements or newsfeed updates to users based on data analysis. Additionally, Twitter uses collected data for real-time query suggestion [30]. Therefore, most solutions exist in the Big Data Application Provider component and should be categorized as Specific Application Layers on Cloud and Mobile IT platforms. Technology vendors such as Oracle [27], IBM [28], and Microsoft [29] have also developed Big Data Reference Architectures [26]. These vendors publish practical Reference Architectures for Big Data toward EA practitioners in corporations and other groups. Most companies already use analytics for forms, reports, and dashboards based on structured data from operational information systems that conform to pre-determined relationships. However, Big Data cannot follow this structured model. The streams are all different and have difficulty establishing common relationships. However, this diversity and abundance can provide opportunities to learn and develop new ideas to support business transformation [24].

The architectural challenge is to bring the above two paradigms together. Therefore, rather than Big Data becoming a new technology silo, organizations should share a unified information architecture to leverage all types of Big Data for promptly satisfying business needs. Oracle provides a practical Big Data Reference Architecture to face the challenges depicted in the Figure 1 of the previous white paper of [24], which provides a conceptual view of the Big Data Analytics Reference Architecture, designed to provide a high-level architecture description of the Big Data and Analytics solution [24].

The above Big Data and Analytics Reference Architecture concentrates the following three important aspects.

- "Unified Information Management" corresponds to the need to manage information holistically, as opposed to governed business silos independently, such as with "High Volume Data Acquisition," which acquires high volumes data with some discards, and "Multi-Structured Data Organization and Discovery," which organizes data of different structures into a common schema.

- "Real-Time Analytics" can contribute to businesses by leveraging information and analysis with prevailing events using "Interactive Dashboards" to react to information and to drill down root cause analyses of situations.

- "Intelligent Processes" can execute business processes more effectively and efficiently, using analysis such as "Optimized Rules," "Recommendations," and "Performance/Strategy Management."

The middle/lower layer of Figure 1 depicted in the previous white paper of [24] represents "Information," in which Big Data and Analytics architecture incorporates many different types of data, such as "Operational Data," "Content," and "Externa1/Analytical data." In the lower layer of Figure 1 depicted in the previous white paper of [24], "Deployment" options are presented for deployment of architecture components, such as "Public Cloud," "Private Cloud," and 
"Managed Services" [24]. Hence, Big Data can be categorized as specific application layers on cloud and mobile IT platforms.

\subsection{EA Frameworks and Analysis - Integrating the elements of Cloud/Mobile IT into EA}

The following descriptions are explanations on the EA frameworks that were the subject of a comparative survey in the previous research paper [23].

\subsubsection{The Open Group Architecture Framework and others}

TOGAF is a framework for the development of EA, which is developed and maintained by the Open Group and includes detailed methods and support tools. The Architecture Development Method (ADM) is the center of TOGAF and is a tiered approach for EA development [9]. TOGAF is most widely used in companies. TOGAF is also discussed from Mobile IT/Cloud/SOA integration perspective [11].

Other EA frameworks are FEAF (Federal Enterprise Architecture Framework 2013), the most widely used framework in government organizations, in particular, DoDAF (Department of Defence Architecture Framework), which is most widely used in the network and security domains, and MODAF (British Ministry of Defence Architecture Framework), which is used by the UK government. These frameworks are discussed by the Federal CIO, the U.S. Department of Defense and Gill from the viewpoint of Mobile IT/Cloud/SOA integration [8, 21, 22]. On the other hand, because the Zachman framework does not provide an enterprise architecture process for implementing and operating an enterprise architecture capability [15], this is also out of our scope at this time.

\subsubsection{Adaptive Enterprise Architecture Framework}

The Adaptive EA framework (also known as the Gill framework) is a meta-framework that enables support by specifying the situation and tailoring an adaptive EA function and framework. It is based on adaptive enterprise service system logic expanding on the SoS (System of Systems) and Agility, service science approach [10]. This adaptive EA framework is defined from the viewpoint of integrating cloud computing elements, and broadly speaking, is composed of the two main layers, namely, external layer (Context, Assessment, Rationalization, Realization, and Un-realization) and internal layer (Defining and others) [11].

Regarding the EA Framework Analysis for integrating the elements of Mobile IT/Cloud into EA, our previous research paper presented the Table 1 to identify the Mobile IT and Cloud computing elements in the EA frameworks selected in this preliminary study [15]. This table should be referred to for detailed understanding of this Analysis. First, to integrate Mobile IT elements, this table in our previous paper shows that Mobile IT related elements such as Mobile devices and API are identified in most of subject EA frameworks. However, no description of Mobile Device Managers or Mobile Application controllers is seen in any subject EA frameworks. Furthermore, to integrate Cloud computing elements, the table in our previous paper shows that many Cloud computing elements, including SaaS, PaaS, and IaaS, are identified in the Adaptive EA framework. Additionally, SaaS, PaaS, and IaaS are seen in the FEAF document IRM and DoDAF documents. However, the description of a Cloud interface is only seen in the Adaptive EA framework. 
According to our previous paper, Mobile IT and Cloud architecture models and guidelines effective in promoting EA are rarely found in the subject EA framework. On the other hand, the Cloud interface, mandatory in hybrid Cloud based implementation important to companies, is only identified in the adaptive EA framework. Based on this prior research and this observation, this EA framework analysis suggests a method to use a framework integrating an adaptive EA framework to support to Cloud elements in corporate entities already implementing EA through applying TOGAF and FEAF, etc. This analysis also suggests the effectiveness of "TOGAF, Mobile IT/Cloud guide" and "Adaptive EA framework Mobile IT/microservice architecture meta-model" if they can be developed [15].

\subsection{Adaptive Integrated EA framework - Aligned with Digital IT Strategy}

Our previous research promoted the strategic use of cloud and mobile IT, suggesting that corporate entities defining Cloud/Mobile IT/Big Data/Digital IT strategies implementing EA by applying frameworks, such as TOGAF and FEAF, could adopt a framework integrating an Adaptive EA framework to provide further support for cloud elements [15]. Accordingly, the preliminary research of this paper proposes an Adaptive Integrated EA framework depicted in Figure 1 of the preliminary research paper, based on this suggestion, which should meet with IT strategy promoting Cloud/Mobile IT/Big Data/Digital IT, and verified this in the case study [23]. The proposed model is an EA framework integrating an adaptive EA cycle with TOGAF or a simple EA framework ${ }^{1}$ for different business units in the upper part of the diagram in [23].

The adaptive EA cycle makes provisions for project plan documents (including architecture designs) for new Digital IT related projects drawn up on a short-term basis. This begins with the Context Phase, which is conducted to prepare the Defining Phase (e.g., architectural design guidelines related to necessary types of Security / Digital IT aligned with IT strategy) per business needs. During the Assessment/Architecture Review Phase, the Architecture Board reviews the architecture in the initiation documents for the IT project. In the Rationalization Phase, the stakeholders and Architecture Board decide upon information systems to be replaced or decommissioned by the proposed new information system. In the Realization Phase, the project team begins to implement the new IT project agreed upon after deliberating issues and action items [23].

The Adaptive EA cycle enables the corporate entity to adopt an EA framework capable of flexibly adapting to new Digital IT projects continuously. Moreover, the TOGAF and simple EA framework, based on an operational division unit in the top part of the Figure 1 of [23], can respond to differing strategies in business divisions in the mid-long-term. This part of the framework has a structure that can select an EA framework based upon the characteristics of division's operational processes and future architectures, while enabling applications. Furthermore, the framework should align EA guiding principles with those business division's principles to keep consistency among the adaptive EA cycle, the TOGAF, and simple EA framework. Furthermore, in the Defining Phase, the Architecture Board promotes the appropriate architectural design of each Digital IT-related system by sharing the architectural guidelines for Security/Digital IT, etc., to align with the IT strategy [23].

\section{Research Methodology}

To investigate Architecture Risks and solutions in ES and EA, we investigate the case study within a large scale GHE, where we built and implemented the "Adaptive Integrated EA 
framework" and started up the Architecture Board. This Global Healthcare Enterprise (GHE) is a research-based global company with its main focus on pharmaceuticals. As the largest pharmaceutical company in Japan and one of the global leaders of the industry, this GHE is committed to strive towards better health for patients worldwide through leading innovation in medicine. This GHE's ethical drugs are marketed in around 100 countries worldwide while the number of this GHE's employees should be approximately 30 thousands. This global pharmaceutical company prioritizes the direction of Digital Healthcare as one of the top element of corporate strategy. Therefore, this global pharmaceutical company need to proceed with digital IT transformation as the high priority and was adopted for the strategic risk mitigation model for digital transformation in this paper. In the global Architecture Board, all new IS/IT project architecture designs were reviewed and action items for the next steps were raised by architects, top managements, and PMO members. After the Architecture Board was held, we defined the risks connected to each action item raised and considered solutions to mitigate them.

As the next step, we propose the strategic risk mitigation model for digital transformation in the Architecture Board as a hypothesis. Furthermore, we analyze the data of activities for digital IT projects in the Architecture Board in this GHE, and we evaluate our proposed model in this empirical research. In our analysis, we mapped solution categories into the previously defined risk categories in the Architecture Board. We analyzed the interrelationships between these risks and solutions that were defined in the architecture risk analysis for digital IT projects involving Big Data related digital IT ones in the Architecture Board.

Based on the above research, we defined the effective strategy elements to mitigate risks with the Architecture Board for EA practitioners as a hypothesis. Moreover, we conducted further research regarding these solutions spreading across the Digital IT Systems, such as Cloud (SaaS, PaaS, and IaaS), Mobile IT applications, and Specific Application Layer on Cloud/Mobile IT(Big Data and Analytics) in this case of GHE. We evaluated our defining elements in the empirical research. Specifically, the research questions employed in this study are:

RQ1: How can the Architecture Board control the Solutions with Risk Mitigation model in Digital Transformation involving Big Data?

RQ2: What Strategy elements can be clarified to mitigate Risks for Digital Transformation?

\section{Architecture Board and Global Healthcare Company Case}

Our preliminary research for this paper proposes an adaptive integrated EA framework to align with IT strategy, promoting Cloud/Mobile IT/Big Data/Digital IT and is verified by our case study [23]. The author of this paper has named the EA framework suitable for the era of Digital IT as "Adaptive Integrated Digital Architecture Framework - AIDAF" at this time. Figure 2 illustrates this AIDAF proposed model. This AIDAF begins with the Context Phase, while referencing the Defining Phase (i.e., architecture design guidelines related to Digital IT aligned with IT strategy). During the assessment and architecture review, the Architecture Board reviews the initiation documents and related architectures for the IT project. 


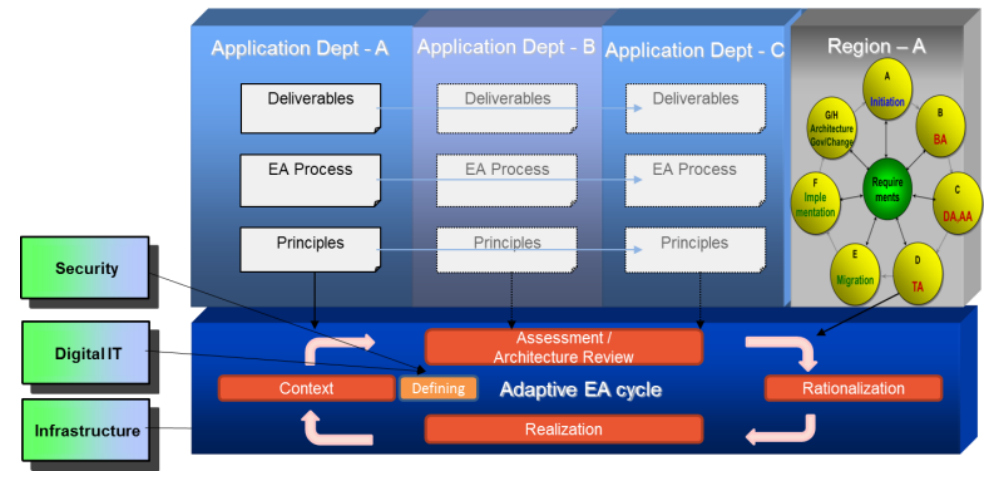

Figure 2. AIDAF proposed model (ex: TOGAF and Adaptive EA framework, etc.)

In a particular Architecture Board, the example of the EA framework structure in a certain global pharmaceutical company examined in this paper is explained. This global pharmaceutical company is the largest Japan-headquartered global company in the industry in Asia based on a sales basis. In a global EA rollout, they are handling Cloud/Mobile IT/Big Data strategic projects and systems that took priority in Europe and US Group companies well by structuring and implementing EA with the above Adaptive Integrated Digital Architecture Framework - AIDAF to be consistent with global IT strategy focusing on Cloud/Mobile IT/Big Data/Digital IT. Table 2: Responsibilities and Tasks of the Architecture Board

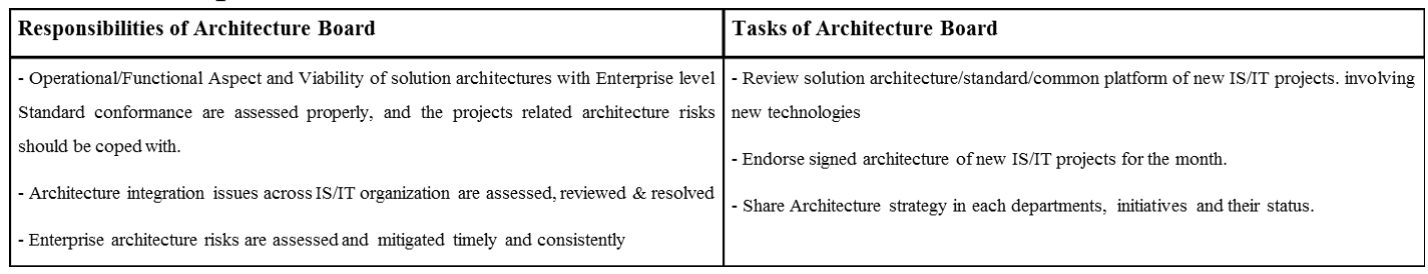

Actually, one of the authors works in this global pharmaceutical company and conducted all phases of structuring and implementing in this EA framework and was the facilitator and managed the coordination of the global Architecture Board. In this global pharmaceutical company, I have the above responsibilities in the Assessment/Architecture Review phase on the global Architecture Board as shown in Table 2, which we are focusing on now, and perform the above tasks. On the other hand, project managers in PMO community propose and brief each new IT project planning documents while I and architecture community members perform reviews and advise their project planning documents and top managements also review and advise (or reject). If the architecture community endorses them, top managements will approve them, and project managers can start these new IT projects. Figure 3 shows the roles and organizations diagram in Architecture Board.

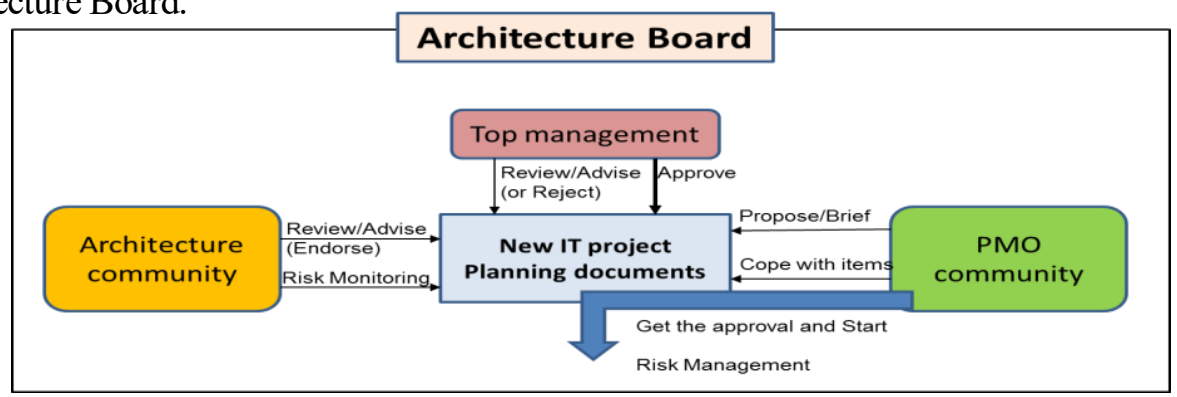

Figure 3. Roles and Organizations diagram in Architecture Board 


\section{Risk Management with Architecture Board}

\subsection{Proposal of STRMM model for Digital Transformation}

As a result of investigating tasks of architecture reviews in the Architecture Board, in this case, we confirmed that project planning documents of almost all new IS/IT projects have been submitted to the Architecture Board. This board performs an Architecture Review on the basis of the following evaluation criteria of 4 elements, namely, [1], [2], [3], and [4] and issued the action items there. The following evaluation criteria of 4 elements are defined in "Activity 4.4: Validate and communicate the conceptual solution architecture" of "Federal Segment Architecture Methodology" [31], as step references for viewpoints of architecture review. One of the authors found out the risks connected to each action item and defined an equivalent solution for each risk, and started the Risk Management process with the Architecture Board based on proper Mitigation Strategy.

[1] Enterprise level conformance - align with architecture roadmap, standard, each architecture principle.

[2] Functional aspect - clarify solutions/architecture specifications, integration points with standard master data.

[3] Operational aspect - alignment with the service level, security design and privacy, system availability.

[4] Viability - application rationalization, risks/countermeasures, data migration/testing strategy.

We propose the "STrategic Risk Mitigation Model for Digital Transformation" in Fig. 4 in this case study as below.

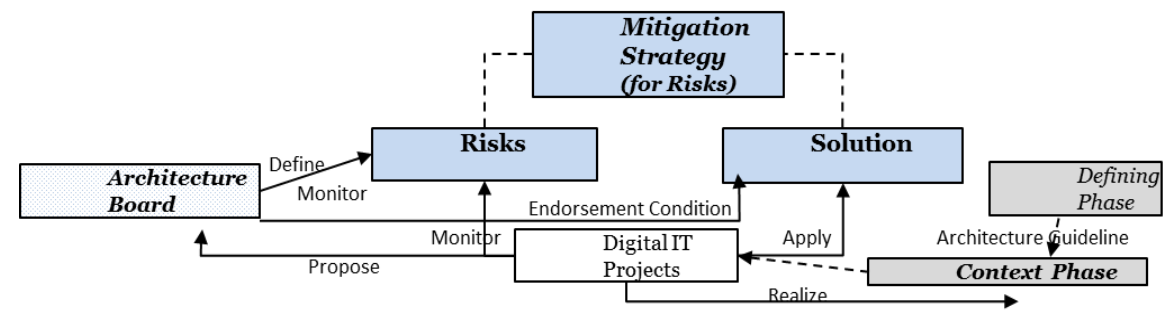

Figure 4: STRMM model for Digital Transformation.

This "STRMM model for Digital Transformation" consists of 7 kinds of entities as Fig. 4 shows. The gray-colored entities show the phase in an Adaptive EA cycle of AIDAF depicted in Fig 2. In the first gray-colored entity of "Context phase" in the Adaptive EA cycle, each project manager in PMO community write down the new IT project planning document for the third entity of "Digital IT project" while referring to Architecture Guidelines developed in the second gray-colored entity of "Defining phase" in the Adaptive EA cycle. As the next step, each project manager can submit and propose the new IT project planning document of "Digital IT project" to the forth entity of the "Architecture Board" as depicted in Fig 4. On the other hand, the blue-colored entities mean the elements raised and defined in Architecture Board reviews. The "Architecture Board" can raise each action item to endorse for the new "Digital IT project" in the reviews, and can define the "Risk" equivalent to each action item, that is the fifth entity of blue-colored one, while the sixth entity of blue-colored "Solution" should be considered/discussed and agreed upon among the Architecture Board members, in consideration with 
Endorsement Conditions, and applied in the "Digital IT project." Furthermore, the seventh entity of blue-colored "Mitigation Strategy element (for Risks)" can be defined in consideration with the relationship between "Risks" and "Solution" as depicted in Fig 4. The new "Digital IT project" will be realized and started after approved by top managements, while Architecture Board and project managers can monitor the status of each "Risk."

\section{Risk/Solution Evaluation for Digital IT areas}

\subsection{Data Analysis - Risk Categories for Digital IT areas}

As a result of data analysis on the results of reviews for Digital IT projects in the Architecture Board in this GHE for 1 year and 8 months, 118 items/risks were raised and categorized into 10 risk categories. The revealed risk categories for Digital IT are presented, with percentages of each category indicated in parentheses.

[1] Security (23\%) - risks mainly related to the information security and privacy, cyber security, security architecture, security solutions, such as user authentication, and access control in digital IT systems.

[2] Architecture Conformance (17\%) - risks mainly connected with solution architecture conformance for digital IT systems in terms of business, process, technical, and sourcing strategy. $11 \%$ of risks are related to Architecture Standard conformance in digital IT area.

[3] Technology Architecture (12\%) - risks mainly connected with technology architecture and target architecture in digital related platforms and mobile applications, digital IT systems, and others.

[4] Project Management (11\%) — risks mainly related to the project management, project scope and cost structure, project scheme and project planning, and project definition.

[5] Compliance and Validation (8.5\%) - risks mainly connected with compliance and validation, such as regulatory compliance and SDLC related compliance, Cloud Data Centers, Cloud Vendors, digital IT related vendors, GxP validation, and testing ones.

[6] Application Architecture (7.5\%) - risks mainly related with application systems architecture and target architecture, roadmap of digital IT systems, in terms of functional aspects and viability.

[6] Data Architecture (7.5\%) - risks mainly connected with data architecture and target architecture, a roadmap of digital IT systems with Master Data Management platforms and data migration strategy.

[6] Application Rationalization (7.5\%) —risks mainly related with application system's rationalization in digital IT areas to prevent from increasing the number of applications in vain.

[9] Strategic Alignment (5\%) - risks mainly connected with the alignment with IT strategy and architecture strategy in digital IT areas and high level of target architecture and roadmap.

[10] System Development (1\%) — risks mainly connected with system development and enterprise system implementations in digital IT areas, and smooth transitioning to the new ES.

\subsection{Data Analysis - Solution Categories for Digital IT areas}

In the Architecture Board in this Global Enterprise, 118 action items with architecture risks have been raised as a result of the architecture reviews for Digital IT projects. Moreover, each solution to mitigate these 118 risks has been defined in the risk assessment process in this global enter- 
prise. During the analysis, we distinguished 10 solution categories for Digital IT areas. These solutions are presented in the following description, which contains short definitions of categories and percentages of adopted solutions as each category. The solution categories for Digital IT areas have been ordered in decreasing order of percentages of the adopted categories. The largest number of category encompasses solutions connected with various approaches for assigning appropriate employees. It is followed by a category that includes solutions related to the involvement of Top Management, such as Global IT Executives, CISO, and regional CIO. Then, with similar frequency of appearance, the Application Architecture and Project Scope Definition categories occur. They are followed by System, Implementation Process, Technology Architecture, and Security Architecture categories. The Architecture Board adopted the least frequent solutions that belong to Vendor Contract Definition and System Vendor categories.

[1] Employees (42\%) - solutions that assign proper employees.

[2] Top Management (18\%) — solutions connected with top management involvement.

[3] Application Architecture (9.5\%) - verification of appropriate application architecture and integration standard.

[4] Project Scope Definition (8\%) - solutions connected with the definition of project scope.

[5] System (7\%) - system customization, modification, and optimization, decommission.

[6] Implementation Process (6\%) — solutions connected with the management of implementation process.

[7] Technology Architecture (4.5\%) - verification of appropriate technology architecture and interface standard.

[8] Security Architecture (4.5\%) — verification of appropriate security architecture and security solution adoption.

[9] Vendor Contract Definition (0.2\%) - solutions connected with vendor contract definition.

[10] System Vendor $(0.1 \%)$ - solutions connected with vendor support and consultants.

\subsection{Interrelation between Solutions and Risks for Digital IT}

Figure 5 presents the mapping of solution categories onto risk categories. In this figure, some solution categories are coped with many risk categories. Specifically, the Employees solution category, which was coped with for risks that belonged to Security, Architecture Conformance, Technology Architecture, Data Architecture and Project Management, Compliance and Validation risk categories. Similarly, solutions from Top Management solution category have been coped with for risks from Architecture Conformance and Data Architecture, Project Management, and Strategic Alignment risk categories. Therefore, the element of "Role Assignments of experts" will be suitable for mitigating risks. 


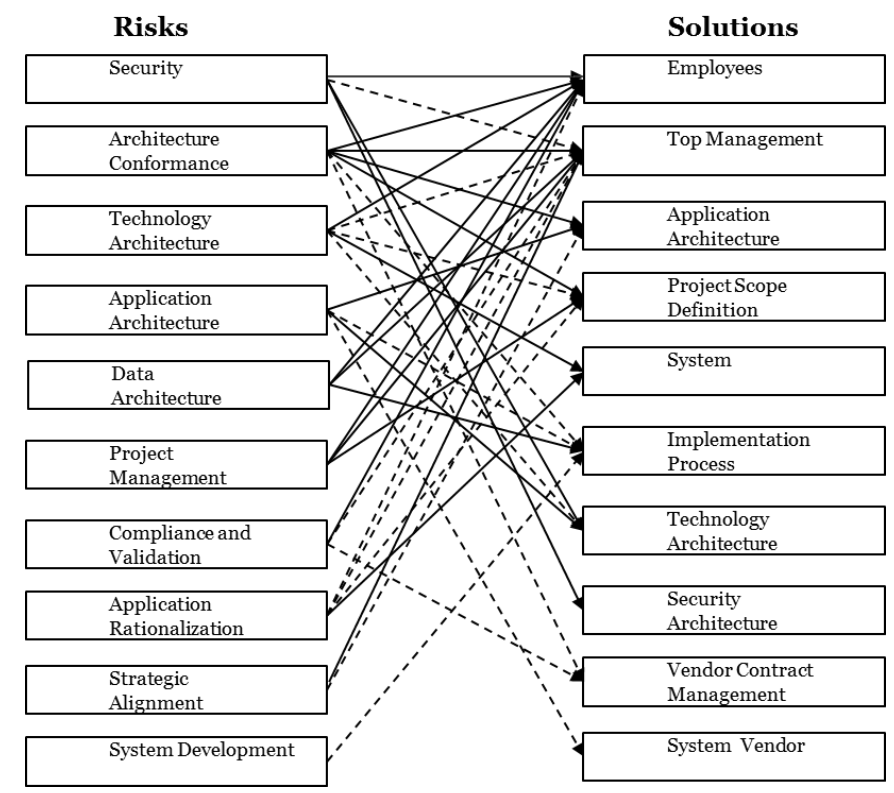

Figure 5: Solution Categories Mapped onto Architecture Risk Categories in Digital IT projects. Note: Solid line denotes direct correspondence between solutions and risk categories more than 3 times. Dotted line shows a direct correspondence between solutions and risk categories more than once.

On the other hand, the Security risk category had been addressed by solutions that belonged to Technology Architecture and Security Architecture categories. For this reason, the element of "Solution Architecting for Security" will contribute to these situations to mitigate risks. Similarly, risks from Application Architecture risk category obtained their solutions from the Application Architecture and Technology Architecture solution categories. Therefore, the element of "Solution Architecting for Application" will contribute to these situations to mitigate risks. Moreover, the Data Architecture risk category had been addressed by solutions that belonged to the Implementation Process solution categories. The element of "Data Architecting" may be suitable for mitigating risks. Furthermore, solutions from the System solution category addressed risks from the Application Rationalization risk category. The element of "System Decommission" should be suitable for mitigating risks. Finally, solutions from Project Scope Definition solution category addressed risks from Project Management risk category. The element of "Project Scoping" should contribute to these situations to mitigate risks.

\section{Discussion}

\subsection{Cloud/Mobile IT with STRMM model}

We proposed the "STrategic Risk Mitigation Model for Digital Transformation" in Fig. 4 for RQ-1. As a result of reviews for Digital IT projects in the Architecture Board in this Global Healthcare Enterprise for 1 year and 8 months, more than 110 kinds of Action Items for new IS/IT projects involving implementations on Cloud, such as SaaS, PaaS, and IaaS, were issued, while 50 kinds of Action Items for new projects involving Mobile IT applications were issued in the Architecture Board.

These new IS/IT projects' documents are developed with reference to Architecture Guidelines and abovementioned Action Items connected to Risks summarized in the aforementioned "Risk Categories." To obtain endorsements for each new IS/IT project involving implementations on 
Cloud from the Architecture Board, the Solutions summarized as "Solution Categories" described in section 6.2 were discussed and defined to mitigate the Risks, and each new IS/IT project has been endorsed/realized by applying these Solutions.

The status of Solutions for each area of Cloud is presented on the left side of Table 2.

First, almost all Solution Categories are defined and controlled for "SaaS Cloud" related projects in the Architecture Board. Extremely high level of solution occurrence is found in the "Security of Employees", and high level of solution occurrences are found in the "EA/Data and Compliance/Validation of Employees", "Strategy of Top Management", "EA/Conformance of Application Architecture", and "Project Management of Project Scope Definition." Moreover, the medium levels of solution occurrence are found in the "Project Management of Employees", "EA/Data and Project Manager of Top Management", "Application Solution of Application Architecture", "System", "Implementation", "Technology Architecture", and "Security Architecture."

Second, half of Solution Categories are defined and controlled for "PaaS Cloud" related projects in the Architecture Board. High level of solution occurrence is found in the "Security of Employees." On the other hand, low level of solution occurrences are found in the Solution Categories of "Employees", "Top Managements", "Application Architecture," etc.

Table 2: Solutions for digital IT areas with mitigation strategy elements

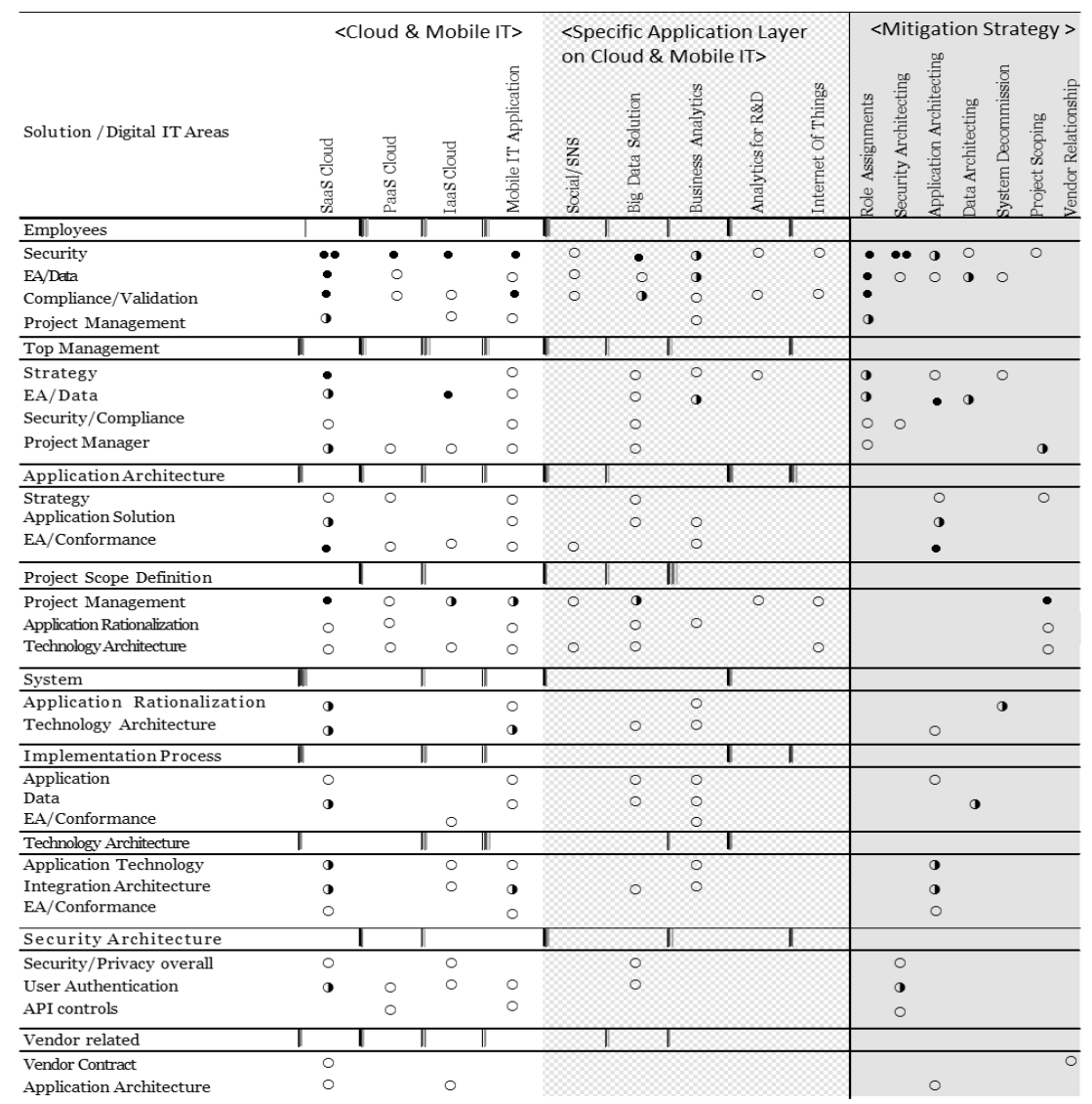


Third, most of Solution Categories are defined and controlled for "IaaS Cloud" related projects in the Architecture Board, except for the "System." High level of solution occurrences is found in the "Security of Employees" and "EA/Data of Top Management." Medium level of solution occurrence is found in "Project Management of Project Scope Definition."

The status of Solutions for the area of Mobile IT Application is shown on the left of Table 2. Almost all Solution Categories are defined and controlled for "Mobile IT Application" related projects in the Architecture Board. High level of solution occurrences is found in the "Security and Compliance/Validation of Employees." Medium levels of solution occurrence are found in the "Project Management of Project Scope Definition", "Technology Architecture of System", and "Integration Architecture of Technology Architecture."

This empirical case study provides evidence how it is possible to manage risks with solutions and strategy elements using the "STRMM model for Digital Transformation" in Fig. 4 in the "Cloud/Mobile IT" areas to cope with RQ1.

\subsection{Specific Application Layer on Cloud/Mobile IT - Big Data with STRMM model}

In the current study, we investigate the specific Application Layer on Cloud and Mobile IT, such as Big Data, social network services, business analytics, analytics for R\&D, and IoT.

The status of solutions for each area of "Specific Application Layer" under "Cloud and Mobile IT" is also presented in the middle (light gray) of Table 2. First, most of the solution categories are defined and controlled for the Big Data solution-related projects, except for "vendor-related solution." In this Global Enterprise, eight kinds of Big Data projects, such as Key Opinion Leaders (KOL), occur. Four kinds of KOL management-related Big Data projects involving mobile IT applications for BI/CRM are raised, all implemented on SaaS cloud, except for one developed on PaaS. Two other kinds of Big Data mobile IT projects were also managed, both implemented on SaaS cloud; one project was implemented on IaaS cloud as well. Another Big Data project was partially-implemented on SaaS and IaaS. The new Big Data Digital IT project documents were proposed and reviewed; 29 action items were raised. High levels of solution occurrence are found in the "Security" under "Employees". Moreover, medium levels of solution occurrences are found in "Compliance/Validation" under "Employees" and "Project Management" under "Project Scope Definition." Moreover, low levels of solution occurrences are found in solution categories under "Employees," "Top Managements," "Application Architecture," "Project Scope Definition," "Implementation Process," and "Security Architecture."

Most of the solution categories are defined and controlled for business analytics-related projects, except for "Security Architecture" and "Vendor-related solution." Medium levels of solution occurrences are found in "Security and EA/Data" under "Employees" and "EA/Data" under "Top Management." Less than three solution categories involving "Employees" and "Project Scope Definition" are defined and controlled for "social/SNS," "analytics for R\&D," and "IoT" projects. The number of the projects in these areas is less than three and limited in this Global Enterprise.

Our current research verifies that the Architecture Board can control solutions with the STRMM model for digital transformation. Table 2 shows the "Specific Application Layer on Cloud and Mobile IT," including "Big Data solution" and "business analytics," to cope with RQ-1. 


\subsection{Strategy elements to mitigate Risks in Digital Transformation}

Based on the research described in Section 6.3, the following strategy risk mitigating elements can be formulated to answer RQ-2. The status of each mitigation strategy element is presented in the right side of Table 2 .

\subsubsection{Role Assignments}

Two types of solution categories, "Employees" and "Top Management," are adopted to mitigate risks with this Strategy element. High levels of solution occurrences are found in "Security," "EA/Data," and "Compliance/Validation" of the "Employees solution category." Moreover, medium levels of solution occurrences are found in "Strategy" and "EA/Data" under "Top Management solution category" and "Project Management" under "Employees solution category." In terms of Big Data projects, high levels of solution occurrence are found in "Security" under "Employees solution category," and medium levels of solution occurrence are found in "Compliance/Validation" under "Employees solution category."

\subsubsection{Security Architecting}

Several kinds of solution categories, including "Employees," "Security Architecture" and "Top Management," are adopted to mitigate risks with this Strategy element. High levels of solution occurrence are found in "Security" under "Employees." Medium levels of solution occurrence are found in "User Authentication" under "Security Architecture." In terms of Big Data projects, high levels of solution occurrence are found in "Security" under "Employees."

\subsubsection{Application Architecting}

Seven kinds of solution categories, including "Employees," "Top Management," "Application Architecture," and "Technology Architecture" are adopted to mitigate risks with this Strategy element. High levels of solution occurrence are found in "EA/Data" under "Top Management" and "EA/Conformance" under "Application Architecture solution." Medium levels of solution occurrence are found in "Security" under "Employees," "Application Solution" under "Application Architecture," and "Application Technology" and "Integration Architecture" under "Technology Architecture."

\subsubsection{Data Architecting}

Three kinds of solution categories, including "Employees," "Top Management", and "Implementation Process" were adopted to mitigate risks with this Strategy element. Medium levels of solution occurrence are found in "EA/Data" under "Employees solution category," "EA/Data" under "Top Management solution," and "Data" under "Implementation Process solution."

\subsubsection{System Decommission}

Three kinds of solution categories, including "Employees," "Top Management," and "System" were adopted to mitigate risks with this Strategy element. Medium levels of solution occurrence are found in "Application Rationalization" under "System solution."

\subsubsection{Project Scoping}


Four kinds of solution categories, including "Top Management," "Employees" and "Project Scope Definition" are adopted to mitigate risks with this Strategy element. High levels of solution occurrence are found in the "Project Management" under "Project Scope Definition," and medium levels of solution occurrence are found in the "Project Manager" under "Top Management solution." For Big Data projects, medium levels of solution occurrences are found in the "Project Management" under "Project Scope Definition."

\subsubsection{Vendor Relationship}

The Vendor-related solution category was adopted to mitigate risks with this Strategy element. However, only low-level solutions are found in the "Vendor Contract."

\subsection{Limitations}

The main limitation of this study relates to the scope of research. Our research was based on data collected from one Global Healthcare Enterprise's Architecture Board reviews. The next limitation of this study relates to the data collection method, researcher bias and human error. One of the authors analyzed the above data collected in the excel sheets on the basis of results of Architecture Board reviews, where experts in each IT category discussed and agreed upon in terms of solutions, while recording their contents, sometimes by hearing, and writing down the minutes of Architecture Board with summarizing action items and risks on the excel sheets.

On the other hand, the actual total number of review results involving the one of risks and solutions raised in the Architecture Board could not be disclosed because of the security policy of this Global Healthcare Enterprise. Furthermore, the explanation of the basis for deriving the formulation of strategy elements to mitigate risks was generalized as the detailed explanation was difficult because of the security policy of this GHE. The number of projects related to "social/SNS," "analytics for R\&D," and "IoT" was limited. Moreover, the scope of the project for "Big Data" was also limited in terms of data architecture, whose data was collected mainly from external social tools and external healthcare IT information sources, while the master data management platform-related project is delayed.

\section{Conclusion}

In this paper, we proposed the "STRMM model for Digital Transformation" as the Risk Mitigation model in the Architecture Board based on the current research with the Global Enterprise case study, where we built and implemented the "Adaptive Integrated Digital Architecture Framework - AIDAF." Based on the empirical data gathered from the result of the Architecture Board reviews in the Global Enterprise, this research verified that the Architecture Board can control the Solutions with "STRMM model for Digital Transformation" in areas of "Cloud/Mobile IT" and "Specific Application Layer on Cloud/Mobile IT", such as "Big Data Solution" to cope with RQ1. Furthermore, we clarified the Strategy elements to mitigate the Risk for Digital Transformation, such as "Role Assignments", "Security Architecting", "Application Architecting", "Data Architecting", "System Decommission", and "Project Scoping", which can lead to the answer for RQ2.

In future research, we would like to the further analyze the solutions for architecture risks covering Security, Application Architecture, Technology Architecture, and Data Architecture that should focus on Digital Healthcare applications related systems, such as the "Internet Of 
Things" raised in the Architecture Board. We aim to propose a new approach to achieve benefits for corporations in these areas.

\section{Acknowledgement}

The authors acknowledge Murli Viswanathan in Carnegie Mellon University Australia for selection of the reference architecture for Big Data.

\section{References}

[1] A. Alwadain, E. Fielt, A. Korthaus, and M. Rosemann, "A comparative analysis of the integration of SOA elements in widely-used enterprise architecture frameworks," Int. J. Intell. Inf. Technol., vol. 9, no. 2, pp. 54-70, 2014.

[2] A. Cruz and A. Vasconcelos, "Architecture for the CRM domain: the Portuguese citizen space case study," Int. J. Enterp. Inf. Syst., vol. 11, no. 2, pp. 24-49, 2015.

[3] S. Boardman and E. Harrington. Open Group Snapshot - Open Platform 3.0 ${ }^{\mathrm{TM}}$. The Open Group, 2015.

[4] S. Buckl, F. Matthes, C. Schulz, and C. M. Schweda, "Exemplifying a framework for interrelating enterprise architecture concerns," in Ontology, Conceptualization and Epistemology for Information Systems, Software Engineering and Service Science, vol. 62, M. A. Sicilia, C. Kop, and F. Sartori, Eds. Berlin, Heidelberg, New York: Springer-Verlag, 2010, pp. 33-46. doi:10.1007/978-3-642-16496-5_3.

[5] H. Chen, R. Kazman, and O. Perry, "From software architecture analysis to service engineering: an empirical study of methodology development for enterprise SOA implementation," IEEE Trans. Serv. Comput., vol. 3, no. 2, pp. 145-160, 2014. doi:10.1109/ TSC.2010.21.

[6] T. H. Davenport, "Putting the enterprise into the enterprise system," Harvard Bus. Rev., vol. 76, no. 4, pp. 121-131, 1998.

[7] B. Familiar, Microservices, IoT and Azure: Leveraging DevOps and Microservice Architecture to Deliver SaaS Solutions. Berkeley, CA: Apress, 2015.

[8] Federal Chief Information Officers Council, A Practical Guide to Federal Service Oriented Architecture, version 1.1., 2008.

[9] J. L. Garnier, J. Bérubé, and R. Hilliard, "Architecture guidance study report 140430," ISO/IEC JTC 1/SC 7 Software and Systems Engineering, 2014.

[10] A. Q. Gill, "Towards the development of an adaptive enterprise service system model," Proceedings of the 19th Americas Conference on Information Systems (AMCIS 2013). Chicago, IL, pp. 15-17, 2013.

[11] A. Q. Gill, S. Smith, G. Beydoun, and V. Sugumaran, "Agile enterprise architecture: a case of a cloud technology-enabled government enterprise transformation," Proceedings of the 19th Pacific Asia Conference on Information Systems (PACIS 2014). United States: AISeL, pp. 1-11, 2014.

[12] A. Q. Gill, “Adaptive cloud enterprise architecture," Intelligent Information Systems, vol. 4. Singapore: World Scientific Publishing Co., 2015.

[13] K. M. Khan and N. M. Gangavarapu, "Addressing cloud computing in enterprise architecture: issues and challenges," Cutter IT J., vol. 22, no. 11, pp. 27-33, 2009.

[14] C. M. MacKenzie, K. Laskey, F. McCabe, P. F. Brown, and R. Metz, "Reference model for service oriented architecture 1.0," Technical Report, Advancing Open Standards for the Information Society, 2006.

[15] Y. Masuda, S. Shirasaka, and S. Yamamoto, "Integrating mobile IT/cloud into enterprise architecture: a comparative analysis," Proceedings of the 21st Pacific Asia Conference on Information Systems (PACIS 2016).Taiwan, paper 4, 2016. 
[16] K. Muhammad and M. N. A. Khan, "Augmenting mobile cloud computing through enterprise architecture: survey paper," Int J Grid Distrib Comput, vol. 8, no. 3, pp. 323336, 2015.

[17] S. Newman, Building Microservices. Sebastopol, CA: O'Reilly Media, Inc., 2015.

[18] A. Qumer and B. Henderson-Sellers, "An evaluation of the degree of agility in six agile methods and its applicability for method engineering," Inf Softw Technol, vol. 50, no. 4, pp. 280-295, 2008.

[19] M. Richards, Microservices vs. Service-Oriented Architecture, 1st ed. Sebastopol, CA: O'Reilly Media, Inc., 2015.

[20] T. Tamm, P. B. Seddon, G. Shanks, and P. Reynolds, "How does enterprise architecture add value to organizations?," Commun Assoc Inf Syst, vol. 28, article 10, 2011.

[21] UK Ministry of Defence, The MODAF Service Oriented Viewpoint, 2010.http://www.mod.uk/DefenceInternet/AboutDefence/CorporatePublications/Infor mationManagement/MODAF/TheServiceOrientedViewpointsov.htm.

[22] US Department of Defense, DoD Architecture Framework Version 2.0., 2009. http://cio-nii.defense.gov/sites/dodaf20/

[23] Masuda, Y., Shirasaka, S., Yamamoto, S. \& Hardjono, T. 201771 : International Journal of Enterprise Information Systems. IGI Global. 13, 3, p. 1-22 22 p. DOI: $\underline{10.4018 / \text { ijeis.2017070101 }}$

[24] D. Chappelle., Big Data \& Analytics Reference Architecture, Oracle Corporation, September 2013.

[25] US Department of Commerce, NIST Big Data Interoperability Framework: Reference Architecture Version 1, 2015.

[26] J. Kein, R Buglak, D Blockow, T Wuttke, "A Reference Architecture for Big Data Systems in the National Security Domain, " the $2^{\text {nd }}$ International Workshop on Big Data Software Engineering, 2016.

[27] Oracle, "Information Management and Big Data." White Paper, 2014, http://www.oracle.com/technetwork/database/bigdataappliance/overview/bigdatarefarc hitecture-2297765.pdf

[28] D. Mysore, S. Khupat, and S. Jain, "Big data architecture and patterns." IBM, White Paper, 2013.

[29] Microsoft, "Microsoft Big Data Solution Brief.", http://download.microsoft.com/ download/F/A/1/FA126D6D-841B-4565-BB26-D2ADD4A28F24/Microsoft Big Data Sol ution_Brief.pdf

[30] P. Pääkkönen, D. Pakkala, "Reference architecture and classification of technologies, products and services for big data systems, “ Big Data Research, 2015 - Elsevier.

[31] C. Coggins, K. Pail, R. VonBostel, "Federal Segment Architecture Methodology Version 1.0.", December 2008. 